\title{
Design of the Clean Room with Eliminating Electrostatic
}

\author{
Yulong Wang ${ }^{1,2, a}$, Dong Zhao ${ }^{2, ~ a ~ a n d ~ L i l i ~} \mathrm{Li}^{1,2, a}$ \\ ${ }^{1}$ Key Laboratory of Engineering Dielectrics and Its Application, Ministry of Education, \\ Harbin University of Science and Technology, Harbin 150080, China; \\ ${ }^{2}$ College of Rongcheng, Harbin University of Science and Technology, Rongcheng 264300, China. \\ ${ }^{a}$ Fly712@126.com
}

Keywords: Eliminate electrostatic, fan filter unit (FFU), high efficiency particulate air filter (HEPA), design

\begin{abstract}
The clean room with eliminating electrostatic was improved to avoid affecting electricity and impurities on properties of materials by tempered glass used as the main framework, electrostatic eliminator and HEPA built in. The effect on eliminating electrostatic and removing dusts was studied by changing size of clean room and air flow based on single variable principle. Analysis results are shown that the size of clean room is $580 \times 580 \times 700 \mathrm{~mm}$; air flow of FFU is $777.024 \mathrm{~m}^{3} / \mathrm{h}$ and size is $570 \times 570 \times 180 \mathrm{~mm}$; HEPA size is $580 \times 580 \times 70 \mathrm{~mm}$ via experimental data and simulation analysis. Electricity and foreign impurities are dislodged in materials by the clean room with eliminating electrostatic. It can be confirmed that the significant impact is formed in the future.
\end{abstract}

\section{Introduction}

With the development of modern society and the progress of science and technology, clean degree of environment is becoming more and more strict for the cable materials in the producting. The research is shown that during rubbed, peeled and extruded materials engender electrostatic charges which result electrostatic adsorption and discharge when the charge accumulates to a certain extent, so that materials contain more impurities and appear easily to patial discharge and break down[1]. The bad influence is formed in terms of electrical properties and mechanical properties.

Many researchers [2] designed the clean room using stainless steel and antistatic curtain to avoid affecting materials properties because of electrostatic and impurities, but their method can't prevent escape of gas effectively, remove static electricity completely and be convenient to movement. In order to avoid these shortcomings, the new clean room without static was designed with tempered glass as main framework, in which static eliminator and HEPA was laid. The new clean room without static can improve efficiency by eliminating internal electrostatic and preventing the escape of gas effectively and has a wide application in the future.

\section{Design Conceptions for Clean Room without Static}

Design conceptions for clean room without static are shown in fig. 1, design of the filter system is shown in fig. 1(a), and design of static electricity removed system is shown in fig. 1(b).

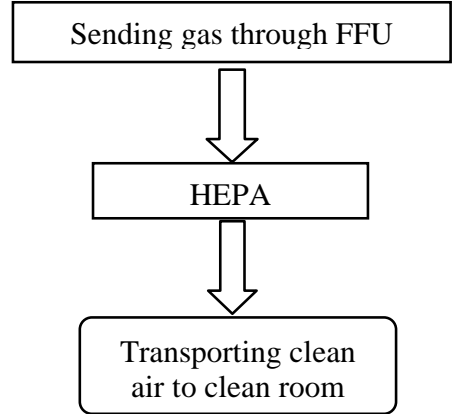

(a) Design of the filter system

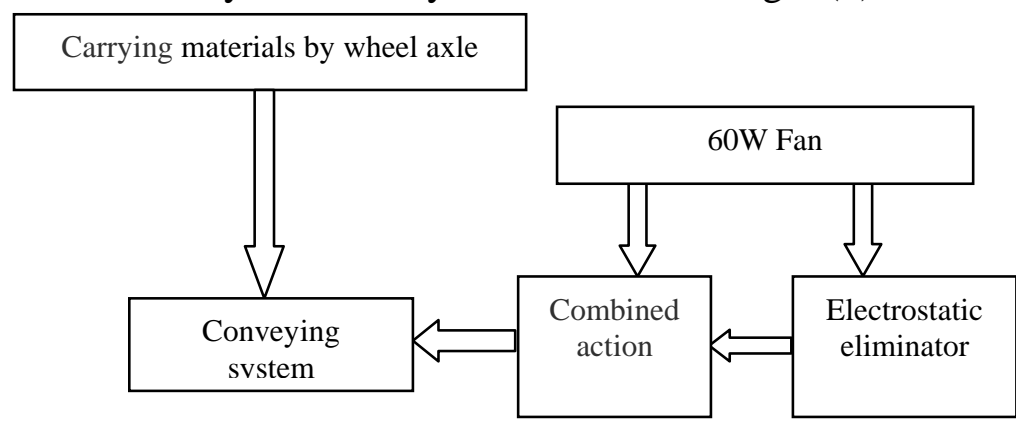

(b) Design of static electricity removed system

Fig. 1 Design conceptions for clean room without static 


\section{Selection of Equipments}

Fan Filter Unit (FFU). The FFU has many advantages, for example good flexibility, adapting to process well and structural simplification and so on [3]. FFU structure is shown in fig. 2. FFU and HEPA adopt split type structure, imported energy saving air blower and diffusion plate, so that the wind speed is uniform and stable. In order to control air flow well, the system has a knob which can regulate wind speed freely [4].
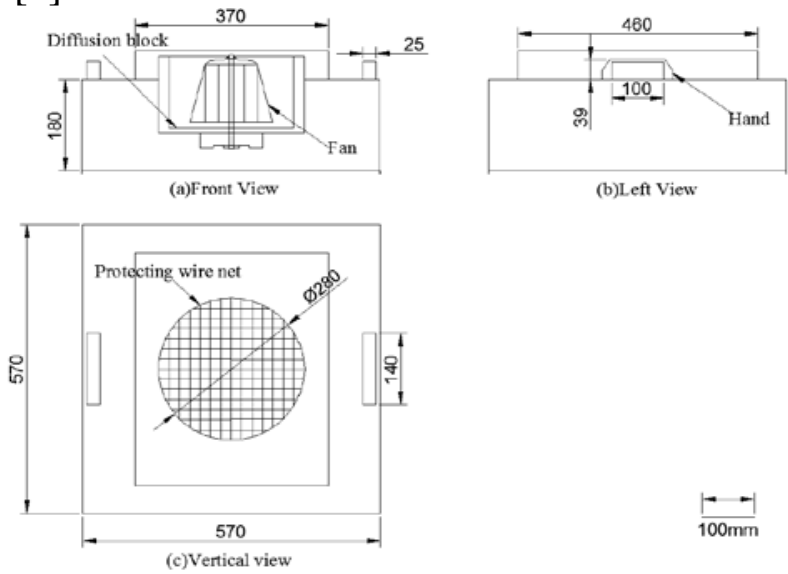

Fig. 2 The structure of FFU

Knob position and wind speed of FFU are determined by air flow, as shown in the formula (1).

$Q=K \times V \times 60\left(\mathrm{~m}^{3} / \mathrm{h}\right)$

$\mathrm{K}$ is generally range from 50 to 60 , which was chose 55 in the article, and the size of the clean room was $580 \times 580 \times 700 \mathrm{~mm}$, so the room volume was $0.23548 \mathrm{~m}^{3}$. the air flow calculated was $777.024 \mathrm{~m}^{3} / \mathrm{h}$.

In this paper, the rated air flow of FFU providing three section speed control is $600-900 \mathrm{~m}^{3} / \mathrm{h}$, which is respectively $0.35 \mathrm{~m} / \mathrm{s} \pm 20 \%, 0.45 \mathrm{~m} / \mathrm{s} \pm 20 \%$ and $0.55 \mathrm{~m} / \mathrm{s} \pm 20 \%[5]$. The knob position chose was $0.45 \mathrm{~m} / \mathrm{s} \pm 20 \%$ after calculating air flow.

High Efficiency Particulate Air Filter(HEPA). The HEPA is mainly composed of filter and shell, the filter is an air filter and the shell is an aluminum alloy shell. Anything don't pass through HEPA, such as the fine particles of $0.1 \mu \mathrm{m}$ and $0.3 \mu \mathrm{m}$.

Experimental results are shown that the characteristic curve of different HEPA is different and that the relationship between drag-flow and air flow can be seen as linear when the actual air flow and the rated air flow is approximate the same, as shown in fig. 3(a). In order to choose the size of HEPA, characteristic curves of drag-flow in fig. 3(b) are synthesized curves in fig. 3(a) (the volume of HEPA $\mathrm{V}$ is $H \times W \times D$ and is seen in table 1 which is specifications of HEPA). It can be seen in fig. 3(b) that curves have the trend of superposition, that is, drag-flow characteristic curve of any HEPA can be roughly known when the actual air flow is not greater than the rated air flow and $Q /(H \times W \times D)$ number in each model is approximately equal at the same initial drag force. In summary, the size of effective filter was $580 \times 580 \times 70 \mathrm{~mm}[7]$.

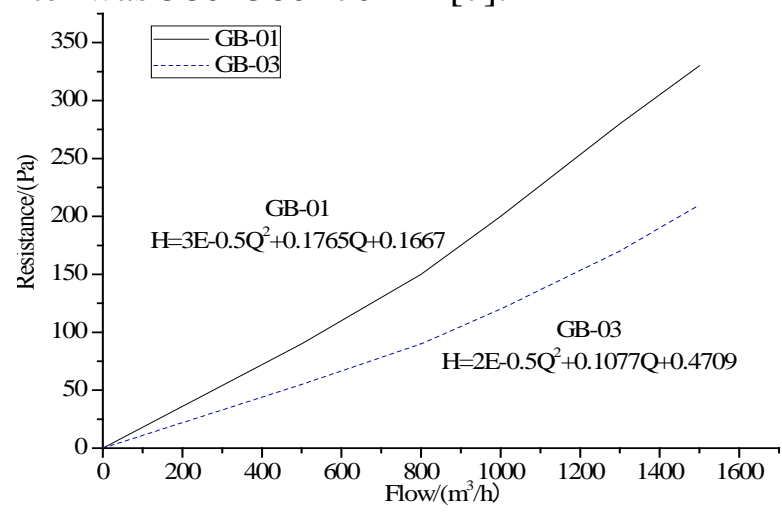

(a) Drag-flow characteristic curve

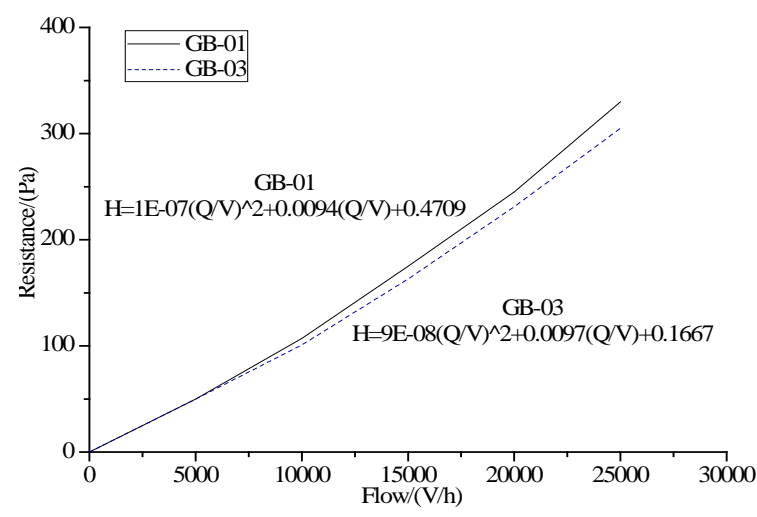

(b) Drag-flow characteristic fitting curve

Fig. 3 Drag-flow characteristic curve of HEPA 
Table 1 Specifications of HEPA

\begin{tabular}{cccc}
\hline Models & $\begin{array}{r}\text { Characteristic dimensions } \\
\mathrm{H} \times \mathrm{W} \times \mathrm{D}(\mathrm{mm})\end{array}$ & $\begin{array}{c}\text { Rated air volume } \\
\mathrm{Q}\left(\mathrm{m}^{3} / \mathrm{h}\right)\end{array}$ & $\begin{array}{c}\mathrm{Q} /(\mathrm{H} \times \mathrm{W} \times \mathrm{D}) \\
\left(\mathrm{h}^{-1}\right)\end{array}$ \\
\hline GB-01 & $484 \times 484 \times 220$ & 1000 & 18182 \\
GB-02 & $484 \times 484 \times 180$ & 800 & 17778 \\
GB-03 & $630 \times 630 \times 220$ & 1500 & 17179 \\
GB-07 & $630 \times 945 \times 220$ & 2250 & 17179 \\
GB-09 & $610 \times 610 \times 150$ & 1000 & 17916 \\
\hline
\end{tabular}

Electrostatic Removed System. Electrostatic is eliminated by the electrostatic eliminator in the clean room with eliminating electrostatic. The SL-001 electrostatic eliminator was chose which was composed of high voltage power supply generator and discharge electrode, and working mechanism is that there is stable high field produced by the tip which brings a large of positive and negative charges in the air to eliminate electrostatic [8]. The working mechanism is shown in fig. 4.

SL-001 electrostatic eliminator has a special ion balance circuit in order to maintain ion balance in high voltage gas. Ion balance circuit is shown in fig. 5 and composed by the polarity reversal circuit, the ion balance adjustment circuit and the high voltage abnormal detection circuit [8].

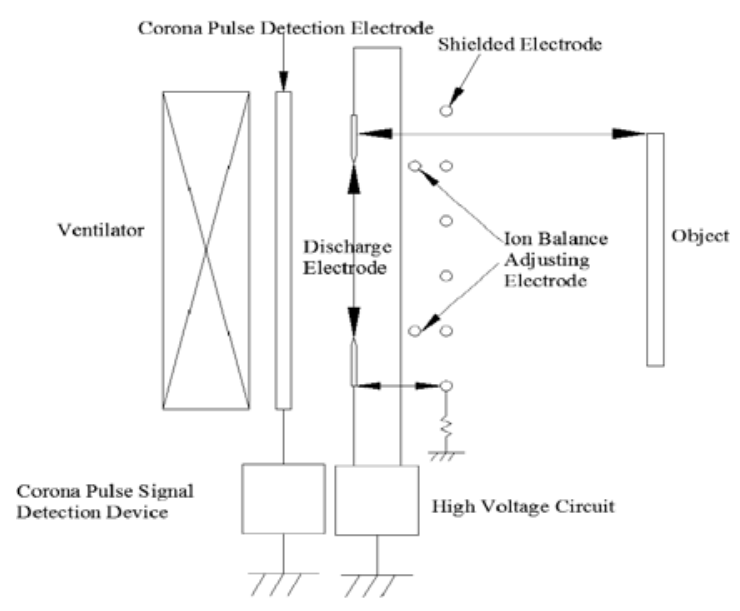

Fig. 4 Working mechanism of electrostatic eliminator

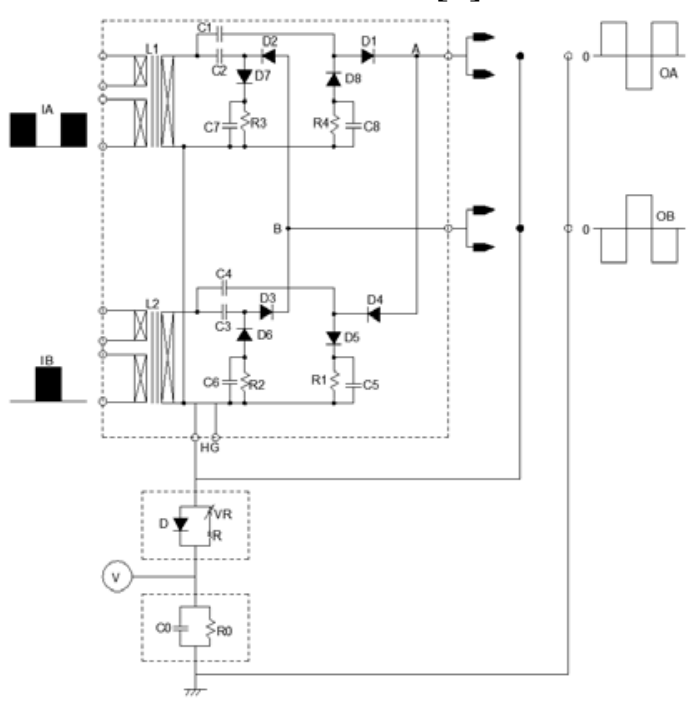

Fig. 5 Ion balance circuit

The polarity reversal circuit is composed by diodes D1-D8, capacitors C1-C8 and resistances R1-R4. In polarity reversal circuit, AC high-voltage changes to DC high-voltage. The output OA is positive voltage and $\mathrm{OB}$ is negative voltage when $\mathrm{IA} \neq 0$ and $\mathrm{IB}=0$, which is the input of reversal circuit. Conversely the output $\mathrm{OA}$ is negative voltage and $\mathrm{OB}$ is positive voltage when $\mathrm{IA}=0$ and $\mathrm{IB} \neq 0$. In summary, the polarity of outputs $\mathrm{OA}$ and $\mathrm{OB}$ arises to reverse at intervals during one cycle and positive and negative ions are exported.

The ion balance adjustment circuit is composed by variable resistance VR, resistance $\mathrm{R}$ and diode D. Ions are balanced by adjusting electric potential of ion balance adjusting electrode which changes concentration of positive and negative ions. The method is that electric potential of ion balance adjusting electrode is changed by changing VR in order to negative ion concentration decrease more rapidly or less slowly[9].

\section{Overall Scheme Design}

The overall design of the clean room with eliminating electrostatic after designing of dimensions and selecting parameters and models of equipments is shown in fig. 6. In fig. 6, the tempering glass is used as the main framework, FFU is installed on the top of the main frame which is used to sending air into the system, HEPA filter is put at the bottom of the FFU which is used to screening air, and the static electricity eliminator is fixed at the end of material conveying system which can produce positive and negative ions to eliminate electricity in materials. 


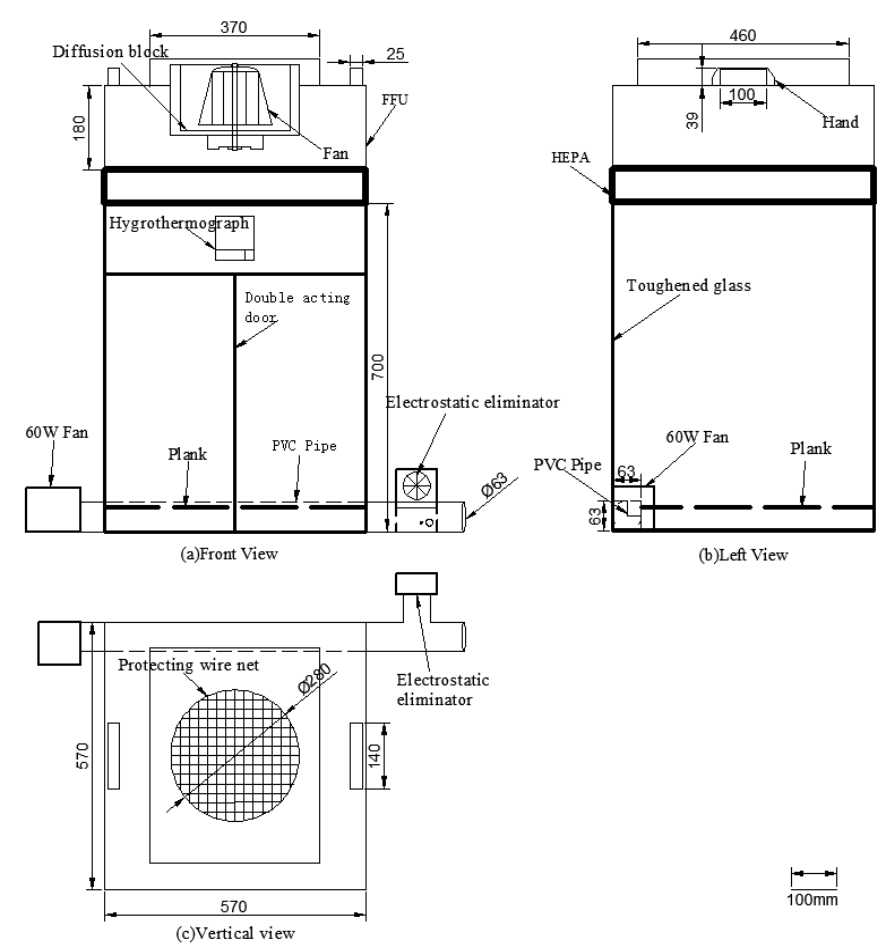

Fig. 6 The overall design of clean room with eliminating electrostatic

\section{Summary}

The clean room with eliminating electrostatic was designed to avoid affecting electricity and impurities on properties of materials. FFU which is the rated air flow $600-900 \mathrm{~m}^{3} / \mathrm{h}$ and three section speed-control, HEPA and SL-001 electrostatic eliminator are chose by means of analyzing mechanisms of eliminating electricity and removing dust, and the size of the clean room is $580 \times 580 \times 700 \mathrm{~mm}$. Electricity and foreign impurities are dislodged in materials by the clean room with eliminating electrostatic, and the quality of materials used can be ensured.

\section{References}

[1] GAO Cheng-de, The Generation and Protection of ESD, Telecom Power Technology. 6 (2009) 60-61.

[2] WEI Jian, The basic technical requirements and test adjustment of the clean room, China Installation Association (Eds.), INSTALLATION, China Installation Association <Installation> periodical office, China, 2012, pp. 26-28.

[3] Zhang Li-qun, Application for FFU, Clean and Air Conditioning Technology. 3 (2003) 46-49.

[4] LIN Zhong-ping, XIAO Xiao-ye, PAN Dong-mei and GAO Da-chun, Performance test research of FFU, BUILDING SCIENCE. 24 (2008) 51-55.

[5] Wang Xiao-xia, the application of FFU air supply mode in a conditioning system, Energy research and utilization. (2005) 40-42.

[6] State Standard of the People's Republic of China, GB 6765-85, Methods for testing the performance of high efficiency particulate air filter-Penetration and drag, Beijing, Standards Press of China, 1985.

[7] LIU Hua, Some analysis of the clean room, BUILDING SCIENCE. 20 (2004) 75-80.

[8] Kazuo Okano, Japan. Patent CN 1930926 A. (2005).

[9] Tomonori Tsumori, Japan. Patent CN 101653046 A. (2010). 